\title{
Bulk matter physics and its future at the Large Hadron Collider
}

\author{
B. Hippolyte ${ }^{\mathrm{a}}$ \\ Institut Pluridisciplinaire Hubert Curien, Département de Recherches Subatomiques and Université Louis Pasteur, Strasbourg, France
}

Received: 7 October 2008 / Revised: 20 January 2009 / Published online: 3 February 2009

(C) Springer-Verlag / Società Italiana di Fisica 2009

\begin{abstract}
Measurements at low transverse momentum will be performed at the LHC for studying particle production mechanisms in $p p$ and heavy-ion collisions. Some of the experimental capabilities for bulk matter physics are presented, focusing on tracking elements and particle identification. In order to anticipate the study of baryon production for both colliding systems at multi-TeV energies, measurements for identified species and recent model extrapolations are discussed. Several mechanisms are expected to compete for hadro-production in the low momentum region. For this reason, experimental observables that could be used for investigating multi-parton interactions and help understanding the "underlying event" content in the first $p p$ collisions at the LHC are also mentioned.
\end{abstract}

PACS 25.75.Dw $\cdot 25.75 .-\mathrm{q}$

\section{Introduction}

A significant physics programme is dedicated to large cross section observables at the LHC. Starting from detector performances and the statistics for the first years of data taking, several items related to bulk matter are discussed in these proceedings. We define here bulk matter physics as the physics dominated by soft processes occurring in heavyion and proton-proton collisions with a specific emphasis on multiple parton interactions. We try to show how hadroproduction at low transverse momentum $\left(p_{\mathrm{T}}\right)$ can be used to investigate non-perturbative QCD phenomena such as quark recombination or, more generally, multi-parton dynamics.

We start with presenting some experimental capabilities at the LHC in the second section: several LHC experiments have tracking devices perfectly suited for identifying low to intermediate $p_{\mathrm{T}}$ (i.e. from $\sim 100 \mathrm{MeV} / c$ to $\sim 6-8 \mathrm{GeV} / c$ ) particles and measuring production yields at mid-rapidity. A few of these detector characteristics are summarized. Due

a e-mail: hippolyt@in2p3.fr to its importance for the soft physics sector, information about the material budget in the central region for ALICE, ATLAS and CMS experiments is indicated as well. The third section deals with baryon production, focusing on different aspects, such as thermal statistical description or baryon number transport. In the fourth section, we concentrate on the hints of multi-parton dynamics, both in $p \bar{p}$ via underlying event studies at CDF as well as intermediate $p_{\mathrm{T}}$ measurements for baryons and mesons in $p p$ and $\mathrm{A}+\mathrm{A}$ collisions at RHIC. Eventually, some model predictions for baryon over meson ratios vs $p_{\mathrm{T}}$ are shown at the nominal LHC energies.

\section{Detector performances for soft physics at the LHC}

Detecting the bulk of particles requires good performances for tracking and identification at low $p_{\mathrm{T}}$. There is also a compromise to be found between a sufficient segmentation for dealing with high multiplicities and a low material budget for having good momentum resolutions. We propose here to check quickly key characteristics related to these issues for some of the LHC experiments.

\subsection{Low $p_{\mathrm{T}}$ performances at mid-rapidity}

Most of the LHC experiments have a broad heavy-ion programme. However, each of them is not equally designed for facing the important multiplicities in $\mathrm{Pb}+\mathrm{Pb}$ collisions at the nominal LHC energies.

At mid-rapidity, the tracking device of the ATLAS experiment consists of a 3-layer pixel barrel followed by a Semi-Conductor Tracker (SCT) strip detector of $4 \times 2$ layers $(|\eta|<2.6)$, and complemented for e/ $\pi$ separation by a Transition Radiation Tracker (TRT) composed of 3 layers of straw-tubes interspersed with a radiator [1]. Charged multiplicity and spectra measurements should benefit from the fine segmentation presented in Table 2.1.

Particle identification via linear energy loss $(d E / d x)$ in the ATLAS central region would rely on a maximum of 11 
Table 2.1 Radius ranges for the subdetectors of the ATLAS barrel tracker and corresponding position resolutions [1]

\begin{tabular}{lll}
\hline Subdetector & Radius range $(\mathrm{cm})$ & Position resolution $(\mu \mathrm{m})$ \\
\hline Pixel & $5-13$ & $10 / 115(\mathrm{r} \phi / \mathrm{z})$ \\
SCT & $30-51$ & $17 / 580(\mathrm{r} \phi / \mathrm{z})$ \\
TRT & $55-109$ & 130 \\
\hline
\end{tabular}

clusters in the silicon layers. It is also important to note that the nominal magnetic field is of $2 \mathrm{~T}$ which makes it challenging for low $p_{\mathrm{T}}$ particles to reach layers at large radius. Eventually, a high occupancy is expected in the TRT already for $p p$ collisions, possibly reaching $90 \%$ for $\mathrm{Pb}+\mathrm{Pb}$ ones (up to $d N_{\mathrm{ch}} / d \eta=3000$ ).

Many studies were performed for anticipating the capabilities of the CMS experiment in the low $p_{\mathrm{T}}$ region and more specifically for heavy-ion collisions $[2,3]$. They mainly involve the central tracker with a pixel detector of 3 layers, a Tracker Inner Barrel (TIB) of double-sided silicon strip sensors and a Tracker Outer Barrel (TOB) of silicon strip detectors in a $4 \mathrm{~T}$ magnetic field. More information on the detector layout can be found in Ref. [4]. It results in an excellent primary vertex and track impact parameter determination together with a maximum occupancy in the tracker of $30 \%$ (layer 4) for $\mathrm{Pb}+\mathrm{Pb}$ [5].

With 10 clusters (where 4 correspond to stereo measurements from the TIB) at mid-rapidity, it was demonstrated that particle identification (PID) via $d E / d x$ can be used to separate successfully charged pions, kaons and protons at low $p_{\mathrm{T}}$. Strange neutral particles are also shown to be identified via invariant mass analyses. A modified algorithm for the pixel detector currently uses a helix parameterization instead of a straight line approximation. Figure 2.1 shows the reconstruction efficiencies as a function of $p_{\mathrm{T}}$ for a charged pion to leave 3 clusters in the pixel detector and being identified with the silicon strip barrels. It contains both efficiencies for minimum bias $p p$ and central $\mathrm{Pb}+\mathrm{Pb}$ collisions as presented in Ref. [2]. Note that a multiplicity of $d N_{\mathrm{ch}} /\left.d y\right|_{y=0}=3200$ was assumed for the central $\mathrm{Pb}+\mathrm{Pb}$ simulations. In the specific case of $p p$ collisions, the average reconstruction efficiencies reach $0.90,0.90$ and 0.86 respectively for pions, kaons and protons with $|\eta|<1.5$. The relative momentum resolution is quite significant at very low $p_{\mathrm{T}}(\sim 10 \%$ for protons at $0.2 \mathrm{GeV} / c)$ but decreases to $6 \%$ already at $1 \mathrm{GeV} / c$.

Tracking in the ALICE experiment is principally based on a large Time Projection Chamber (TPC) and an Inner Tracking System (ITS) using three different silicon technologies: pixel, drift and strip detectors (2 layers for each, respectively named SPD, SDD and SSD). There is a full azimuthal coverage and the pseudorapidity range for a charged particle is $|\eta|<0.9$ [6]. The experiment was originally designed for central $\mathrm{Pb}+\mathrm{Pb}$ multiplicities up to $d N_{\mathrm{ch}} / d \eta=$

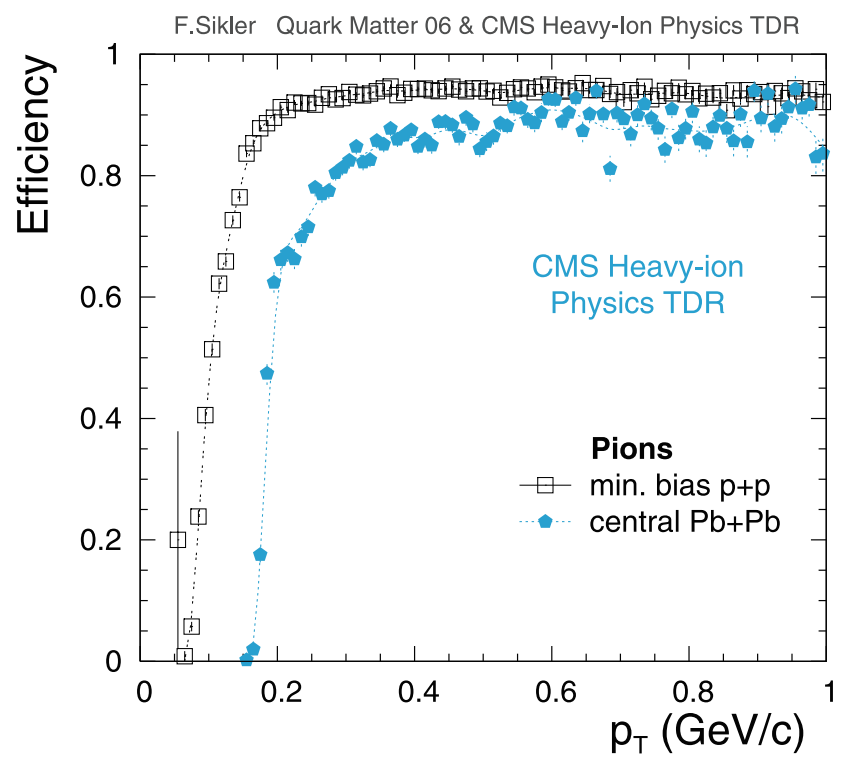

Fig. 2.1 Reconstruction efficiency as a function of $p_{\mathrm{T}}$ for pions using the CMS silicon tracker and within the pseudorapidity range $|\eta|<1$. Both efficiencies for minimum bias $p p$ events (open squares) and for central $\mathrm{Pb}+\mathrm{Pb}$ collisions (full circles) are represented [2]

8000. It has an excellent efficiency and resolution at low $p_{\mathrm{T}}$ benefiting from a moderate magnetic field of $0.5 \mathrm{~T}$.

The TPC $d E / d x$ resolution is below $6 \%$ and $8 \%$ respectively for $p p$ and $\mathrm{Pb}+\mathrm{Pb}$ collisions in the $p_{\mathrm{T}}$ range of [0.2-1.5] GeV/c with tracks having between 90 and 150 clusters. Many other detectors will contribute to PID in a wide $p_{\mathrm{T}}$ range and not only for charged particles (for instance via Ring Imaging Cherenkov, Time of flight determination or with a Transition-Radiation Detector for electron identification) but also for some neutral particles (e.g. via secondary vertex reconstruction and invariant mass analysis). Detailed estimates for PID ranges and efficiencies can be found in Ref. [7], such as Fig. 2.2 which illustrates the very good efficiency obtained for different combinations of ALICE detectors at mid-rapidity, for both minimum bias $p p$ and central $\mathrm{Pb}+\mathrm{Pb}$ collisions.

\subsection{Material budget}

Cumulative material budget has a very important influence for low $p_{\mathrm{T}}$ measurements since it impacts on absorption and multiple scattering. A summary table, including ALICE, ATLAS and CMS information, is available in Ref. [8] and indicates that ALICE tracker is the thinnest with $13 \%$ of radiation length (i.e. approximately the third of the ATLAS or CMS one). In Fig. 2.3, we show the material distribution (in radiation length) of the ALICE central tracking region up to the TPC (outer radius of $2.6 \mathrm{~m}$ ) and averaged over the azimuthal angle $\phi$.

A logarithmic scale is chosen for distinguishing properly the different contributions of the ITS subdetectors as well as 


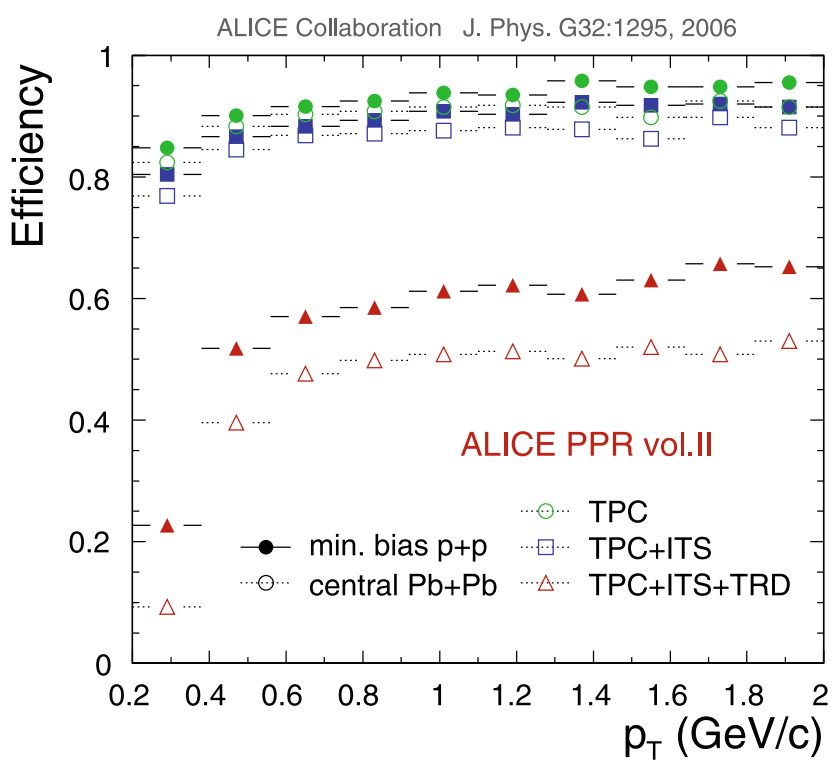

Fig. 2.2 Track-finding efficiency with different combinations of ALICE detectors at mid-rapidity for minimum bias $p p$ (full symbols) and central $\mathrm{Pb}+\mathrm{Pb}$ (open symbols) collisions $\left(d N_{\mathrm{ch}} / d \eta=6000\right)$ [7]

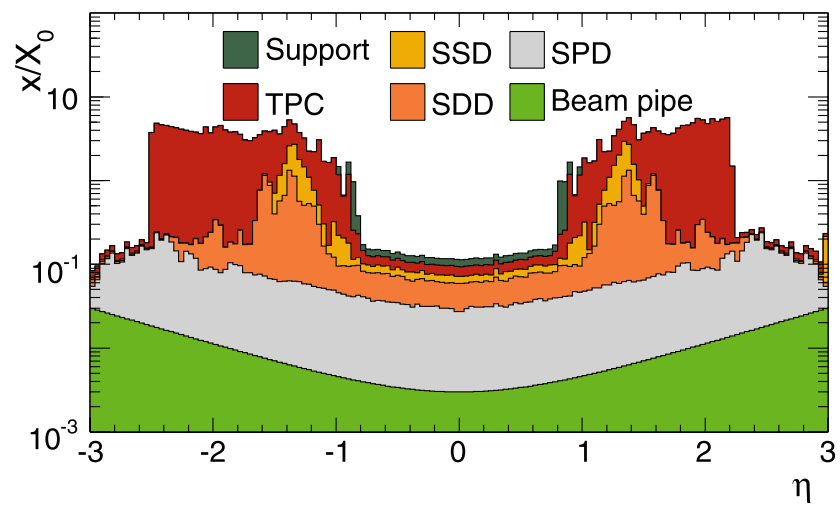

Fig. 2.3 Material distribution (in radiation length $X_{0}$ ) of the ALICE central tracking region, averaged over the azimuthal angle $\phi$. Contributions of individual subdetectors include service and support. Details of cumulative values at mid-rapidity and comparison with other LHC experiments can be found in Ref. [8]

the TPC in the central region. Equivalent figures for ATLAS and CMS can be found in Refs. [1, 4].

\section{Baryon production and asymmetry studies}

Statistical thermal models reproduce quite successfully hadron yields over a large energy range. Therefore several extrapolations at LHC energies are already available for $\mathrm{Pb}+\mathrm{Pb}$ collisions, anticipating a baryo-chemical potential very close to zero (see Ref. [9] and references therein). For $p p$ collisions, one can even try to distinguish between different correlated volumes within the canonical description [10].

At the LHC, having a vanishing net-baryon density and a large gap between beam rapidities create ideal conditions for studying baryon number transport. This can be done by measuring an asymmetry between baryon and anti-baryon production at mid-rapidity. Predictions for such an asymmetry vary significantly, depending on models, when including or not baryonic string junctions [11]. The experimental challenge is then to have sufficiently low systematical uncertainties for being able to draw conclusions [12]. It must be noted, however, that measurements consistent with no asymmetry were recently reported [13].

\section{Multi-parton dynamics in $p p$ and $\mathrm{Pb}+\mathrm{Pb}$}

In this section, we present briefly how multiple parton interactions were investigated by studying the underlying event structure in $p+\bar{p}$ with the CDF experiment at the Tevatron and how this can be pursued with PID for $p p$ collisions at the LHC. Then basic features of the quark recombination are summarized with an illustration showing the possible interplay between coalescence and fragmentation in the intermediate $p_{\mathrm{T}}$ region.

\subsection{Underlying event studies}

In $p p$ or $p \bar{p}$ at high energy, the emphasis is usually laid on the hard 2-to-2 parton-parton scattering with a high transverse momentum and fragmenting into jets of particles. However a significant fraction of particle production is coming from the "underlying event", defined as the beamremnants plus initial and final state gluon radiations as shown on the left side of Fig. 4.1.

Disentangling the different contributions can be complicated both phenomenologically and experimentally [14] but specific measurements from the CDF experiment gave insights on this issue [15]. The idea is to distinguish several angular regions dominated by each contribution. Hence their definition is with respect to the back-to-back main jet direction as shown in the right side of Fig. 4.1: the "towardside" region corresponds to leading (charged) jet direction with $|\Delta \phi|<60^{\circ}$ whereas the "away-side" region is defined by $|\Delta \phi|>120^{\circ}$. Complementarily, the "transverse" region is given by the interval $|\Delta \phi| \in\left[60^{\circ}-120^{\circ}\right]$ and is the most sensitive to the "underlying event" and its soft structure.

Reproducing the main features of both contributions simultaneously is not an easy task for QCD Monte Carlo models since the underlying structure is simply not a minimum bias event. It triggered developments implying soft or semisoft multiple parton interactions in order to reproduce the 

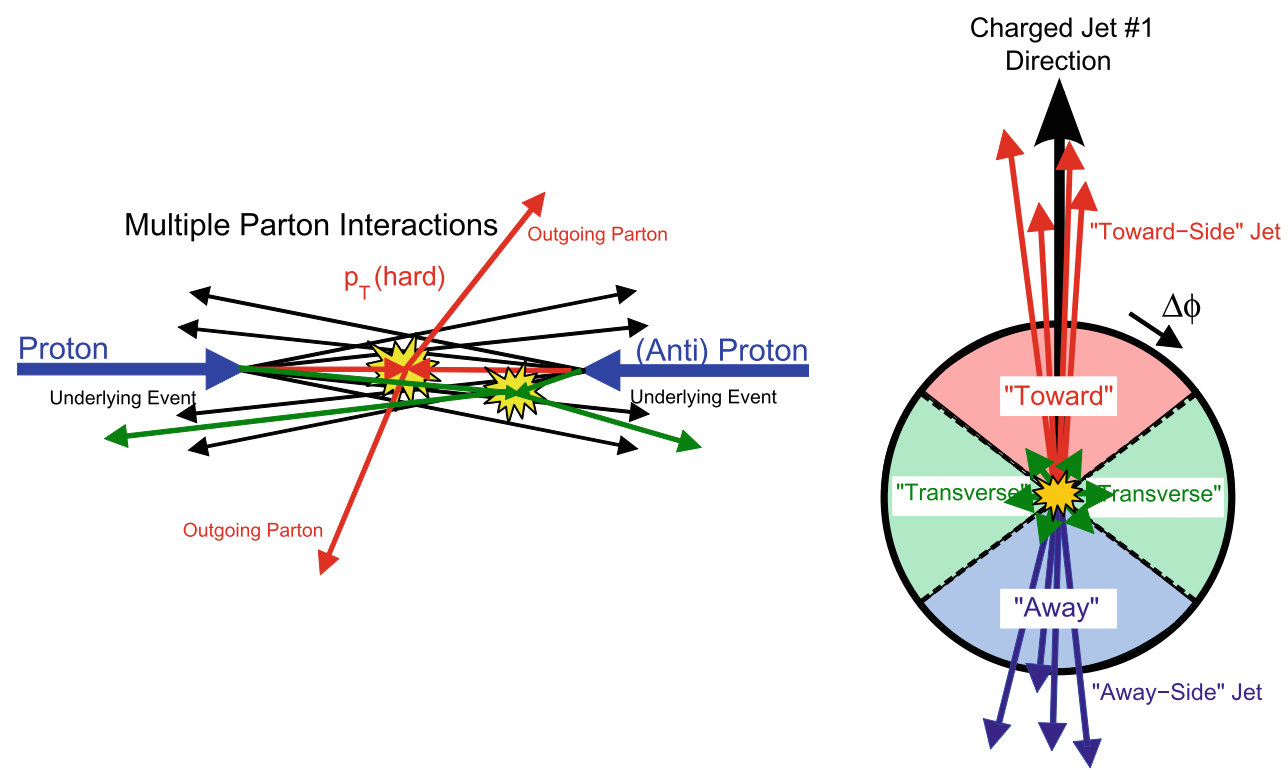

Fig. 4.1 Left: Illustration of the "underlying event" modeled by PYTHIA for $p \bar{p}$ collisions by including multiple parton interactions (together with the hard 2-to-2 parton-parton scattering with a transverse momentum $p_{\mathrm{T}}$-hard, there is a second "semi-hard" 2-to-2 partonparton scattering which contributes to particle production). Right: Illus- tration of the correlations in the azimuthal angle relative to the direction of the leading charged jet noted as "Charged Jet \#1" in the event. The "transverse" region, which is perpendicular to the axis of the hard 2to-2 scattering, is very sensitive to the "underlying event" component of the QCD Monte Carlo models [14]
Fig. 4.2 Illustration of the possible competition between fragmentation and coalescence in the intermediate $p_{\mathrm{T}}$ range with: a) corresponds to a high $p_{\mathrm{T}}$ quark fragmentation into a meson, whereas $b$ ) and $c$ ) show respectively the creation of a meson and a baryon via quark recombination

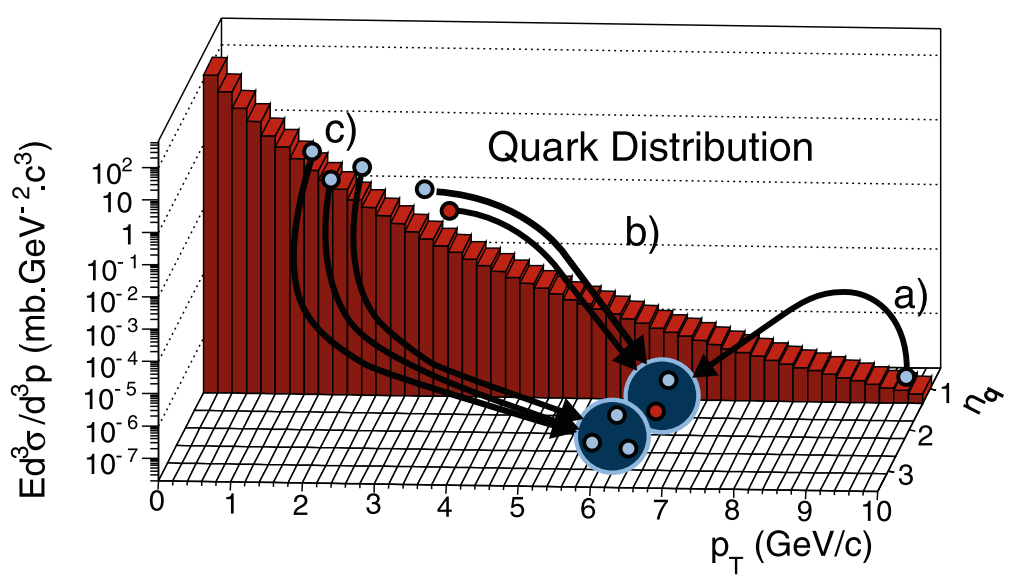

charged particle multiplicity or the transverse mean $p_{\mathrm{T}}[16]$. More constraints could be given using at the same time PID and a lower $p_{\mathrm{T}}$ cut at the LHC energies.

\subsection{Coalescence and hadro-production at RHIC}

By going from $p p$ to $\mathrm{A}+\mathrm{A}$ collisions at a sufficiently high energy in the center of mass, it is expected to switch from a dilute system of partons with high virtualities to a dense one with low virtualities eventually equilibrating to form a Quark Gluon Plasma. If the phase space is filled with partons, hadronization via recombination/coalescence can be envisaged under "the assumption of a thermalized parton phase" [17]. In the intermediate $p_{\mathrm{T}}$ region, in vacuo frag- mentation competes with in medium recombination of lower momentum quarks as shown in Fig. 4.2. It illustrates the fact that due to a quickly decreasing quark momentum distribution, making a $6 \mathrm{GeV} / c$ pion from the recombination of two quarks at $3 \mathrm{GeV} / c$ is favoured with respect to the fragmentation of a $10 \mathrm{GeV} / c$ parton. For the same reason, such a production mechanism was invoked to explain the high baryon over meson ratios in the transverse momentum range between 1 and $5 \mathrm{GeV} / c$ which were observed at RHIC as well as constituent quark scaling. Developments including higher Fock states in order to take into account sea quarks and gluons and not only valence quarks can be found in Ref. [18]. 


\section{Baryon/meson ratios in $p p$ collisions at the $\mathrm{LHC}$}

\subsection{Reference for heavy-ion collisions}

As reminded in Sect. 4.2, the baryon over meson (B/M) ratios measured at RHIC are considered as a first step for investigating recombination and coalescence mechanisms. A summary of the measurements which were performed by the STAR experiment can be found in Ref. [19]. Probing the differences between baryon and meson spectrum shapes in $\mathrm{A}+\mathrm{A}$ at the $\mathrm{LHC}$ requires PID over a large $p_{\mathrm{T}}$ range. Calculations for the LHC are already available assuming a transverse radial flow extrapolation: the amplitude for mixed ratios are predicted to be comparable to the RHIC ones but the turnover should be shifted to slightly higher $p_{\mathrm{T}}$ values [20]. However, with the first LHC data being that of elementary $p p$ collisions, it also means that very detailed studies must be performed similarly for this colliding system as Ref. [9].

\subsection{Predictions from PYTHIA and EPOS}

We report several extrapolations for $p p$ collisions at the LHC nominal energy of $14 \mathrm{TeV}$. Using PYTHIA v6.214, we start comparing the baryon production for a standard minimum bias extrapolation tuned by the ATLAS Collaboration and three underlying event descriptions. The descriptions only differ here by the $p_{T \text { min }}$ value used in PYTHIA and below which the perturbative parton-parton hard scattering cross section is assumed to vanish. The value is chosen be proportional to the square of the centre-of-mass energy to a chosen power $\epsilon$ [21].

In Fig. 5.1, we show the results of these PYTHIA simulations in performing the ratio between the baryon production from the underlying event ("und. event") and minimum bias ("ATLAS tune") as a function of $p_{\mathrm{T}}$. Both $p+\bar{p}$ and $\Lambda+\bar{\Lambda}$ are represented (respectively dashed lines and symbols). With a decreasing $p_{T \text { min }}$ (corresponding to a decreasing power $2 \times \epsilon$ ), the possible contribution of the underlying event increases exactly in the intermediate $p_{\mathrm{T}}$ region. It indicates that the underlying event contribution can reach more than twice the one of minimum bias event at the top LHC energies. Note must be taken that no $s / u$ flavour dependence is observed, making the strange baryon interesting to study such an effect (topology identification of strange baryon decays is efficient in this $p_{\mathrm{T}}$ domain).

In Fig. 5.2, we compare extrapolations at LHC energies for minimum bias $p p$ obtained with PYTHIA and the EPOS model which uses parton-based Regge theory [22] and directly includes quantum mechanical multiple scattering $[23,24]$. The tune used for PYTHIA v.6.3 was already described in Ref. [9] and references therein. The extrapolation for EPOS at LHC energies is exactly the one reported in Ref. [25]. The presented ratios $(\Lambda+\bar{\Lambda}) / \mathrm{K}$ as a function
Transverse momentum ratios: ATLAS Tune vs Und. event

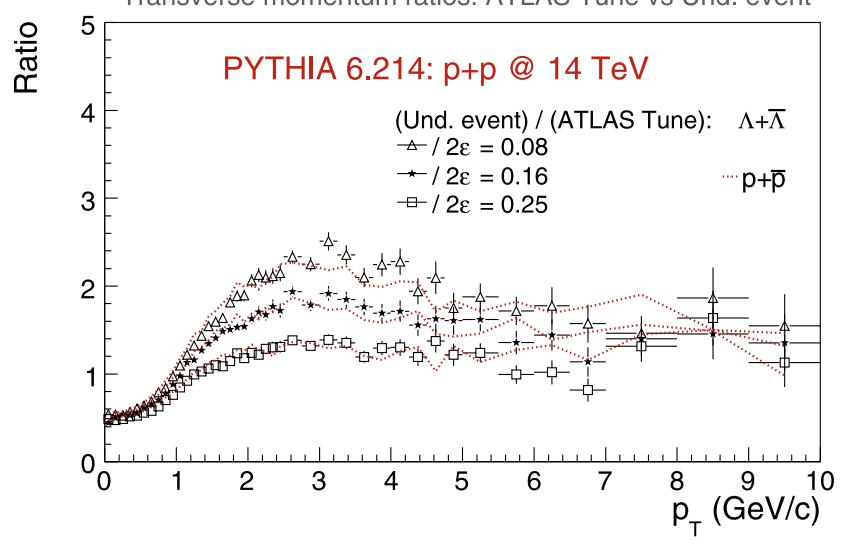

Fig. 5.1 Ratio of baryon productions in $p p$ collisions at $14 \mathrm{TeV}$ in the centre of mass, for $\Lambda$ 's (symbols) and protons (dashed lines) between PYTHIA ATLAS tune and underlying event descriptions: no $s / u$ flavour dependence is observed for these PYTHIA simulations and the underlying event contribution may be quite significant in the intermediate $p_{\mathrm{T}}$ region at LHC energies

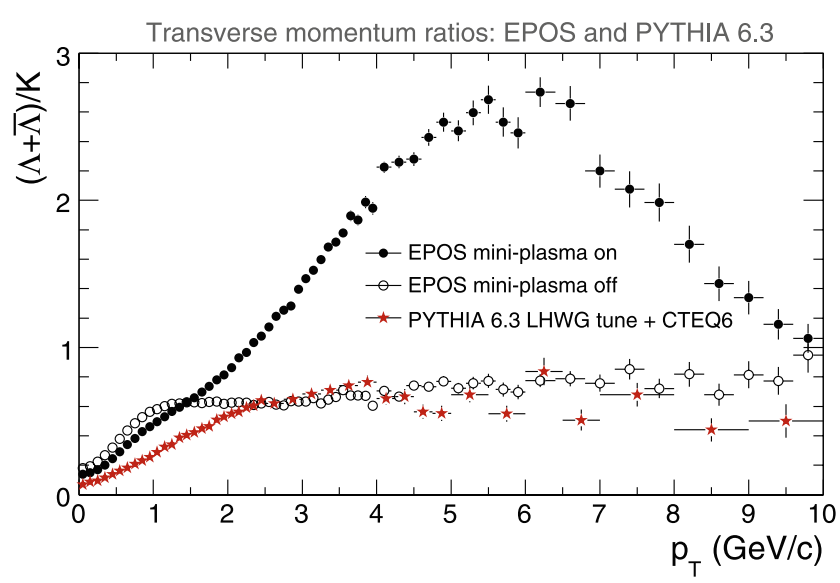

Fig. 5.2 Comparison of single strange baryon over meson ratio $(\Lambda+\bar{\Lambda}) / \mathrm{K}$ at LHC top energies for $p p$ collisions and simulated with PYTHIA and EPOS models. Two configurations are used for EPOS, corresponding to the mini-plasma option being respectively on (full circles) and off (open circles) [25]

of $p_{\mathrm{T}}$ differ slightly (less than a $5 \%$ effect on the overall $p_{\mathrm{T}}$ range) because one corresponds to neutral kaons (PYTHIA) whereas the other is for charged kaons (EPOS). The possible occurrence of mini-plasma cores leading to collective effects already in $p p$ is illustrated by the "mini-plasma on" option: it would give a very significant baryon production with respect to meson ones for $p_{\mathrm{T}}$ between 2 and $9 \mathrm{GeV} / c$.

\section{Summary}

We presented some detector elements and their characteristics relevant for bulk matter physics at the LHC energies. To anticipate hadro-production in $p p$ collisions at low 
transverse momentum, we reported several extrapolations mainly based on multiple parton interactions. In particular, it was discussed how particle identification can help defining the structure of the underlying event in $p p$, and which hadronization mechanisms are expected to compete in $\mathrm{A}+\mathrm{A}$ collisions in the intermediate transverse momentum region. Eventually specificities of baryon and meson productions at the top LHC energies were shown based on PYTHIA and EPOS models.

\section{References}

1. S. Bentvelsen et al. (ATLAS Collaboration), JINST 3, S08003 (2008)

2. F. Sikler (CMS Collaboration), Int. J. Mod. Phys. E 16, 1819 (2007)

3. F. Sikler (CMS Collaboration), arXiv:0805.0809

4. R. Adolphi et al. (CMS Collaboration), JINST 3, S08004 (2008)

5. D.G. d'Enterria et al. (CMS Collaboration), J. Phys. G 34, 2307 (2007)

6. K. Aamodt et al. (ALICE Collaboration), JINST 3, S08002 (2008)

7. B. Alessandro et al. (ALICE Collaboration), J. Phys. G 32, 1295 (2006)
8. J.-P. Revol, Nucl. Phys. B 177-178, 60 (2008)

9. B. Hippolyte (ALICE Collaboration), Eur. Phys. J. C 49, 121 (2007)

10. I. Kraus, J. Cleymans, H. Oeschler, K. Redlich, arXiv:0808.0611

11. L. Montanet, G.C. Rossi, G. Veneziano, Phys. Rep. 63, 149 (1980)

12. P. Christakoglou, M. Oldenburg, ALICE Note 2008-010

13. A. Falkiewicz (H1 Collaboration), Presented at Deep Inelastic Scattering Workshop, London, 2008

14. R. Field (CDF Collaboration), Presented at "From HERA and TEVATRON to LHC", Madrid, FERMILAB-CONF-06-530-E, 2006

15. A.A. Affolder et al. (CDF Collaboration), Phys. Rev. D 65, 092002 (2002)

16. T. Sjöstrand, P. Skands, Eur. Phys. J. C 39, 129 (2005)

17. R.J. Fries et al., Phys. Rev. C 68, 044902 (2003)

18. B. Müller, R.J. Fries, S.A. Bass, Phys. Lett. B 618, 77 (2005)

19. Y. Xu (STAR Collaboration), These proceedings

20. R.J. Fries, B. Müller, Eur. Phys. J. C 34, S279 (2004)

21. T. Sjöstrand et al., Comput. Phys. Commun. 82, 74 (1994). hep-ph/0308153

22. H.J. Drescher et al., Phys. Rep. 350, 93 (2001)

23. K. Werner, F.M. Liu, T. Pierog, Phys. Rev. C 74, 044902 (2006)

24. S. Portebœuf, These proceedings

25. N. Armesto et al., J. Phys. G: Nucl. Part. Phys. 35, 054001 (2008) 